\title{
BERGMAN COMPLETENESS IS NOT A QUASI-CONFORMAL INVARIANT
}

\author{
XU WANG \\ (Communicated by Franc Forstneric)
}

\begin{abstract}
We show that Bergman completeness is not a quasi-conformal invariant for general Riemann surfaces.
\end{abstract}

Recently, B.-Y. Chen (see [Chen1]) asked a question about whether Bergman completeness is a quasi-conformal invariant of Riemann surfaces. In this paper we will give an example showing that the answer to the question is negative.

The original idea of the example is based on the paper [Wwo1. In Zwo1, the growth of the Bergman kernel near the boundary has been estimated with the help of potential-theoretical quantities. Also in [Ju, a necessary and sufficient condition for Bergman completeness of Zalcman type domains has been found. The present paper will use the methods in Pfl-Zwo.

For $0<r<\frac{1}{4}$ and $t \in\left(0, \frac{1}{2}\right)$ define

$$
A^{r, t}:=\bigcup_{k=1}^{\infty} A_{k}^{r, t} \cup\{0\},
$$

where

$$
A_{k}^{r, t}:=\left\{r^{k} e^{i \theta}:-2 \alpha_{k} \leq \theta \leq 2 \alpha_{k}\right\}
$$

and $\sin \alpha_{k}=e^{-t^{-k}}$.

Finally we put $D^{r, t}:=\triangle(0,1) \backslash A^{r, t}$, where $\triangle(p, r):=\{z \in \mathbb{C}:|z-p|<r\}$, $p \in \mathbb{C}, r>0$.

\section{Theorem 1.}

(1) $D^{r, t}$ is Bergman exhaustive if and only if $r^{2} \leq t$;

(2) if $t \leq r^{4}$, then $D^{r, t}$ is not Bergman complete.

Corollary 2. Bergman completeness is not in general a quasi-conformal invariant for Riemann surfaces.

Recall that a homeomorphism $f$ defined on a domain in the complex plane is called $L(L>1)$ quasi-conformal if it is differentiable almost everywhere and

$$
\left|\frac{\partial f}{\partial \bar{z}}\right| \leq \frac{L-1}{L+1}\left|\frac{\partial f}{\partial z}\right|
$$

Received by the editors May 12, 2011 and, in revised form, June 28, 2011.

2010 Mathematics Subject Classification. Primary 32F45; Secondary 32A25.

The author would like to thank W. Zwonek for his fruitful suggestions on this paper.

This project operated within the Foundation for Polish Science IPP Programme "Geometry and Topology in Physical Models", co-financed by the EU European Regional Development Fund, Operational Program Innovative Economy 2007-2013. 
Before we present the proofs of Theorem 1 and Corollary 2 let us recall some notions and results which we need in the paper.

In order to prove Theorem 1, we need some lemmas on logarithmic capacity and Bergman kernel (see Jar-Pf] $)$. First we recall necessary notions from potential theory (see [Ran, where also other properties of potential-theoretic objects that we use are given).

For a probabilistic measure $\mu$ defined on all Borel subsets of a compact set $K$ (denote $\mu \in \mathcal{P}(K)$ ), we define its logarithmic potential by

$$
p_{\mu}(z):=\int_{K} \log |z-w| d \mu(w), z \in \mathbb{C} .
$$

Recall that $p_{\mu}$ is harmonic in $\mathbb{C} \backslash K$ and subharmonic in $\mathbb{C}$.

We also denote the energy of $\mu$ as follows:

$$
I(\mu):=\int_{K} p_{\mu}(z) d \mu(z)=\int_{K} \int_{K} \log |w-z| d \mu(w) d \mu(z) .
$$

A probabilistic measure $\nu$ defined on Borel subsets of the compact set $K$ is called the equilibrium measure of $K$ if $I(\nu)=\sup \{I(\mu): \mu \in \mathcal{P}(K)\}$. It is well known that the equilibrium measure exists and is unique if $K$ is not polar (a set $F \subset \mathbb{C}$ is called polar if there is a subharmonic function $u$ defined on $\mathbb{C}$ such that $u \not \equiv-\infty$ and $F \subset\{u=-\infty\}$ ).

The logarithmic capacity of a subset $E$ of $\mathbb{C}$ is given by the formula

$$
\operatorname{cap}(E):=e^{\sup \{I(\mu): \mu \in \mathcal{P}(K), K \text { is a compact subset of } E\}} .
$$

In the case when $K$ is compact and not polar, then $\operatorname{cap}(K)=e^{I(\nu)}$, where $\nu$ denotes the equilibrium measure of $K$. It is well-known that a Borel set $E \subset \mathbb{C}$ is polar iff $\operatorname{cap}(E)=0$.

Now let us recall the notion of the Bergman kernel. Let $D$ be a bounded domain in $\mathbb{C}^{n}$. Denote by $L_{h}^{2}(D)$ square integrable holomorphic functions on $D$. $L_{h}^{2}(D)$ is a Hilbert space with the scalar product induced from $L^{2}(D)$ denoted by $\langle\cdot, \cdot\rangle_{D}$ (we also denote by $\|\cdot\|_{D}$ the $L^{2}$ norm on $D$, and the space of square-integrable holomorphic functions on $D$ is denoted by $\left.L_{h}^{2}(D)\right)$.

Let us define the Bergman kernel of $D$ as

$$
K_{D}(z)=\sup \left\{\frac{|f(z)|^{2}}{\|f\|_{D}^{2}}: f \in L_{h}^{2}(D), f \not \equiv 0\right\}, z \in D
$$

and the fundamental form of the Bergman metric as

$$
B_{D}(z)=i \partial \bar{\partial} \log K_{D}(z), z \in D \text {. }
$$

Let

$$
I_{D}(z, X)=\sup \left\{\frac{\left|f^{\prime}(z) X\right|^{2}}{\|f\|_{D}^{2}}: f \in L_{h}^{2}(D), f(z)=0, f \not \equiv 0\right\}, z \in D, X \in \mathbb{C}^{n} .
$$

The following result is classical:

$$
B_{D}(z)(X)=\frac{I_{D}(z, X)}{K_{D}(z)}, z \in D, X \in \mathbb{C}^{n} .
$$

Let $D$ be a bounded domain in $\mathbb{C}^{n}, z_{0} \in \partial D$. Then $D$ is Bergman exhaustive at $z_{0} \in \partial D$ if $\lim _{D \ni z \rightarrow z_{0}} K_{D}(z)=\infty$. We call $D$ Bergman exhaustive if $D$ is Bergman exhaustive at $z_{0}$ for any $z_{0} \in \partial D$. 
A bounded domain $D$ is called Bergman complete if any Cauchy sequence with respect to the Bergman distance is convergent to some point in $D$ under the standard topology of $D$. For references on the Bergman kernel, metric and distance, see Chen2.

It is known that if a bounded domain $D \subset \mathbb{C}$ is Bergman exhaustive, then $D$ is Bergman complete (see Chen3); the converse implication does not hold in general (see [wo2]). Also a bounded hyperconvex domain is Bergman exhaustive (see Ohs $)$ and Bergman complete (see [B-P, [Her]).

Now let us introduce the notions necessary for the description of Bergman exhaustive points in dimension one.

For a bounded domain $D \subset \mathbb{C}$ and for a point $z \in \bar{D}$, we introduce the following potential-theoretic quantity:

$$
\gamma_{D}^{(n)}(z):=\int_{0}^{1 / 4} \frac{d \delta}{\delta^{2 n+3}(-\log (\operatorname{cap}(\bar{\triangle}(z, \delta) \backslash D))} .
$$

The following lemma comes from the paper [Zwo1].

Lemma 3. Let $D$ be a bounded domain in $\mathbb{C}$ and let $z_{0} \in \partial D$. Then

$$
\lim _{D \ni z \rightarrow z_{0}} \gamma_{D}^{(0)}(z)=\infty
$$

if and only if

$$
D \text { is Bergman exhaustive at } z_{0} \text {. }
$$

Remark 4. It also follows from classical results that the domain $D^{r, t}$ is Bergman exhaustive at all of its boundary point except for 0 .

Since we shall only consider bounded domains in $\mathbb{C}$, for simplicity we denote $\beta_{D}(z)=B_{D}(z)(1), z \in D$. From the paper [Pfl-Zwo], we know

Lemma 5. Let $D$ be a bounded domain in $\mathbb{C}, D \ni z_{k} \rightarrow z_{0} \in \partial D$. If

$$
\limsup _{k \rightarrow \infty} \gamma_{D}^{(1)}\left(z_{k}\right)<\infty
$$

then

$$
\limsup _{k \rightarrow \infty} \beta_{D}\left(z_{k}\right)<\infty .
$$

Now we move to the proofs of the main results. Let us first see how we derive Corollary 2 from Theorem 1. Then we show Theorem 1.

Proof of Corollary 2. For $\alpha>\frac{1}{2}$ we define

$$
\varphi(z)=z^{\alpha} \bar{z}^{\alpha-1} \text {. }
$$

Note that $\varphi$ is a quasi-conformal mapping from $D^{r, t}$ to $D^{r^{2 \alpha-1}, t}$. Choosing for instance $r=1 / 8, \alpha=2 / 3, t=1 / 32$ we get that Bergman completeness is not a quasi-conformal invariant. Actually $D^{r, t}$ is, in view of Theorem 1 (1), Bergman exhaustive and thus it is Bergman complete, whereas $D^{r^{2 \alpha-1}, t}$ is, in view of Theorem 1 (2), not Bergman complete. 
Remark 6. By Theorem 1 we also can deduce that Bergman exhaustiveness is not a quasi-conformal invariant. But we still do not know whether it is a conformal invariant for bounded domains in the complex plane. Also due to the above theorem, there are lots of domains which are Bergman complete but not Bergman exhaustive (see [Wo2]).

Note also that the example from Corollary 2 is a Riemann surface with infinitedimensional fundamental group. But B.-Y. Chen informed the author that Bergman completeness is a quasi-conformal invariant when the fundamental group is finitely generated.

Behind Chen's question on the quasi-conformal invariance of the Bergman completeness, there is an affirmative result by Pfluger on the quasi-conformal invariance of the existence of the Green function and a very deep negative result by BeurlingAhlfors on the invariance of the nullity of linear measure for subsets of the circle as the boundary of the disc (see [Sa-Nak]). The author would like to thank the referee for this comment.

Proof of Theorem 1. If $D^{r, t}$ is Bergman exhaustive, then by Lemma 3,

$$
\lim _{D^{r, t} \ni z \rightarrow 0} \gamma_{D^{r, t}}^{(0)}(z)=\infty
$$

For any $\delta \in[0,1 / 4]$, if $-1<x<0$ and $|x|$ is small enough, we have

$$
\bar{\triangle}(x, \delta) \backslash D^{r, t} \subset \bar{\triangle}(0, \delta) \backslash D^{r, t} .
$$

Thus for such an $x$,

$$
\gamma_{D^{r, t}}^{(n)}(x) \leq \gamma_{D^{r, t}}^{(n)}(0)
$$

consequently, we get $\gamma_{D^{r, t}}^{(0)}(0)=\infty$. Now choose

$$
\begin{gathered}
A_{1}=\left\{z \in \mathbb{C} ; 2 r^{2} \leq|z| \leq 1 / 4\right\}, \\
A_{k}=\left\{z \in \mathbb{C} ; 2 r^{k+1} \leq|z| \leq 2 r^{k}\right\}, k \geq 2 .
\end{gathered}
$$

We have

$$
\gamma_{D^{r, t}}^{(n)}(0)=\left(\int_{2 r^{2}}^{1 / 4}+\sum_{k=2}^{\infty} \int_{2 r^{k+1}}^{2 r^{k}}\right) \frac{d \delta}{\delta^{2 n+3}\left(-\log \left(\operatorname{cap}\left(\bar{\triangle}(0, \delta) \backslash D^{r, t}\right)\right)\right.} .
$$

Let

$$
\begin{gathered}
C_{1}=\int_{2 r^{2}}^{1 / 4} \frac{d \delta}{\delta^{2 n+3}\left(-\log \left(\operatorname{cap}\left(\bar{\triangle}(0, \delta) \backslash D^{r, t}\right)\right)\right.}, \\
C_{k}=\int_{2 r^{k+1}}^{2 r^{k}} \frac{d \delta}{\delta^{2 n+3}\left(-\log \left(\operatorname{cap}\left(\bar{\triangle}(0, \delta) \backslash D^{r, t}\right)\right)\right.}, k \geq 2 .
\end{gathered}
$$


Then we have

$$
\begin{gathered}
\left(1 / 4-2 r^{2}\right) 4^{2 n+3} \frac{1}{-\log \left(\operatorname{cap}\left(A_{2} \backslash D^{r, t}\right)\right)} \leq C_{1}, \\
C_{1} \leq\left(1 / 4-2 r^{2}\right)\left(2 r^{2}\right)^{-2 n-3} \sum_{j=1}^{\infty} \frac{1}{-\log \left(\operatorname{cap}\left(A_{j} \backslash D^{r, t}\right)\right)}, \\
\left(2 r^{k}\right)^{-2 n-2}(1-r) \frac{1}{-\log \left(\operatorname{cap}\left(A_{k+1} \backslash D^{r, t}\right)\right)} \leq C_{k}, \\
C_{k} \leq\left(2 r^{k+1}\right)^{-2 n-2}(1 / r-1) \sum_{j=k}^{\infty} \frac{1}{-\log \left(\operatorname{cap}\left(A_{j} \backslash D^{r, t}\right)\right)}, k \geq 2 .
\end{gathered}
$$

As $\operatorname{cap}\left(A_{j} \backslash D^{r, t}\right)=r^{j} e^{-t^{-j}}$, we get

$$
\frac{t^{j}}{1-t \log r} \leq \frac{1}{-\log \left(\operatorname{cap}\left(A_{j} \backslash D^{r, t}\right)\right)} \leq t^{j}
$$

Thus there is a sufficiently large constant $C(t, r, n)$ such that

$$
C(t, r, n)^{-1}\left(\frac{t}{r^{2 n+2}}\right)^{k} \leq C_{k} \leq C(t, r, n)\left(\frac{t}{r^{2 n+2}}\right)^{k}
$$

Now due to the lower semi-continuity of $\gamma_{D^{r}}^{(0)}([$ Zwo1] $)$, the theorem follows easily from Lemma 3 (part (1)) and Lemma 6 (part (2)).

\section{REFERENCES}

[B-P] Z. Błocki and P. Pflug, Hyperconvexity and Bergman completeness, Nagoya Math. J. 151 (1998), 221-225. MR1650305 (2000b:32065)

[Chen1] B.-Y. Chen, An essay on Bergman completeness, preprint, 2011.

[Chen2] B.-Y. Chen, A remark on the Bergman completeness, Complex Variables Theory Appl. 42, no. 1 (2000), 11-15. MR1786123 (2001e:32049)

[Chen3] B.-Y. Chen, Completeness of the Bergman kernel on non-smooth pseudoconvex domains, Ann. Polon. Math. LXXI(3) (1999), 242-251. MR.1704301 (2000i:32021)

[Her] G. Herbort, The Bergman metric on hyperconvex domains, Math. Z. 232(1) (1999), 183-196. MR 1714284(2000i:32020)

[Jar-Pfl] M. Jarnicki and P. Pflug, Invariant Distances and Metrics in Complex Analysis, Walter de Gruyter, Berlin, 1993. MR 1242120 (94k:32039)

[Ju] P. Jucha, Bergman completeness of Zalcman type domains, Studia Math. 163 (2004), 71-83. MR2047465 (2005b:32025)

[Ohs] T. Ohsawa, On the Bergman kernel of hyperconvex domains, Nagoya Math. J. 129 (1993), 43-52. MR.1210002 (93k:32049)

[Pfl-Zwo] P. Pflug and W. Zwonek, Logarithmic capacity and Bergman functions, Arch. Math. (Basel) 80 (2003), 536-552. MR 1995634 (2004m:30012)

[Ran] T. Ransford, Potential Theory in the Complex Plane, Cambridge University Press, 1995. MR:1334766 (96e:31001)

[Sa-Nak] L. Sario and M. Nakai, Classification Theory of Riemann Surfaces, Springer, 1970. MR0264064(41:8660) 
[Zwo1] W. Zwonek, Wiener's type criterion for Bergman exhaustiveness, Bull. Polish Acad. Sci. Math. 50 (2002), 297-311. MR.1948078 (2003k:30016)

[Zwo2] W. Zwonek, An example concerning Bergman completeness, Nagoya Math. J. 164 (2001), 89-102. MR1869096 (2002i:32010)

Instytut Matematyki, Uniwersytet Jagielloński, Łojasiewicza 6, 30-348 Kraków, Poland

Current address: Department of Mathematics, Tongji University, Shanghai 200092, People's Republic of China

E-mail address: 1113xuwang@tongji.edu.cn 